\title{
Genome-wide RAD sequencing to identify a sex-specific marker in Chinese giant salamander Andrias davidianus
}

\author{
Qiaomu Hu ${ }^{1 *}$, Cuifang Chang ${ }^{2}$, Quanhe Wang ${ }^{1,3}$, Haifeng Tian ${ }^{1}$, Zhigang Qiao ${ }^{4}$, Lei Wang ${ }^{4}$, Yan Meng ${ }^{1}$, \\ Cunshuan $\mathrm{Xu}^{2^{*}}$ and Hanbing Xiao ${ }^{1 *}$
}

\begin{abstract}
Background: Chinese giant salamander Andrias davidianus is an endangered species. The success of artificial breeding provides a useful way to protect this species. However, the method to identify the sex and mechanism of sex determination were unclear which hinder the improvement of the artificial breeding. Detection of a sex specific marker provides an effective approach to identify genetic sex and investigate the sex determination mechanism.

Results: We used restriction-site-associated DNA (RAD) sequencing to isolate a sex-specific genetic marker in $A$. davidianus to expand knowledge of the sex determination mechanism. Four male and four female specimens were subjected to RAD sequencing, which generated 934,072,989 reads containing approximately $134.4 \mathrm{~Gb}$ of sequences. The first round of comparison of the assembled sequence against the opposite sex raw reads revealed 19,097 female and 17,994 male unmatched sequences. Subsequently, 19,097 female sequences were subjected to a BLAST search against male genomic data, which revealed 308 sequences unmapped to the male genome. One hundred of these were randomly selected and validated by PCR in five male and five female specimens, and four putative sex-specific sequences were produced. Further validation was performed by PCR in another 24 females and 24 males, and all female individuals exhibited the expected specific bands, while the males did not. To apply the sexspecific marker, three specimens reversed from genetic female to physiological male were found in a group exposed to elevated temperature, and 13 individuals reversed from genetic male to physiological female were obtained in a 17ß-estradiol exposed group.
\end{abstract}

Conclusion: This is the first report of a sex-specific marker in A. davidianus and may have potential for elucidation of its sex determination mechanism and, hence, its conservation.

Keywords: Andrias davidianus, RAD-seq, Female-specific marker, Sex identification, Sex reversal

\section{Background}

Genetic mechanisms, including sex determination, are highly variable among vertebrates. Genetic sex determination can be either male heterogamety $(\mathrm{XX} / \mathrm{XY})$ or female heterogamety $(\mathrm{ZZ} / \mathrm{ZW})[1,2]$. To understand the fundamental biological processes of sex determination and sex reversal, the identification of sex chromosomes

\footnotetext{
* Correspondence: hqmu0806@163.com; cellkeylab@126.com; xhb@yfi.ac.cn ${ }^{1}$ Yangtze River Fisheries Research Institute, Chinese Academy of Fishery Sciences, Wuhan 430223, Hubei, China

${ }^{2}$ State Key Laboratory Cultivation Base for Cell Differentiation Regulation, College of Life Science, Henan Normal University, Xinxiang 453007, Henan Province, China

Full list of author information is available at the end of the article
}

is necessary. Sex manipulation in breeding made possible by identification of the sex chromosomes can have considerable economic value $[3,4]$.

Several techniques can be employed to identify chromosomal sex. Most simply, the karyotype is visualized by cytogenetic techniques to detect the heteromorphic sex chromosome [5-7]. Breeding of sex reversed neomales and neofemales can reveal which sex is heteromorphic based on the sex ratio of the progeny [8-12]. In toad Bufo bufo, a sex reversed female mated with the normal male produced all male progeny, indicating male homogametic sex determination mechanism (ZZ) and female heterogametic (ZW) [8]. Gynogenesis

(C) The Author(s). 2019 Open Access This article is distributed under the terms of the Creative Commons Attribution 4.0 International License (http://creativecommons.org/licenses/by/4.0/), which permits unrestricted use, distribution, and 
uses heterogenous sperm or inactivated sperm to activate eggs, which are then heat-shocked to prevent the second meiotic division, essentially producing female self-fertilization. This technique yields all female progeny, indicating the $\mathrm{XX} / \mathrm{XY}$ system in the studied species [13-17].

Each of these techniques presents an associated challenge. The karyotype is not available for fish, amphibians, and reptiles that possess a telocentric chromosome or lack the heteromorphic sex chromosome [18, 19]. Both producing a sex reversed individual in many species as well as producing sufficient numbers of progeny to analyze the sex ratio to infer the genetic type are challenge. Development of a sex-specific marker shows excellent potential for identification of the sex chromosome in various species.

A sex-specific marker is especially valuable in species that lack distinguishable sexually dimorphic phenotypes and in specimens at early stages of development that lack secondary sex characteristics. The most common means of investigating a sex-specific marker involves amplified fragment length polymorphism (AFLP) [3, 20] or microsatellites [21, 22]. However, the effort involved in these methods is enormous and these approaches cannot be applied for all of the species. Additionally, if any restriction enzyme failed to identify a sex-specific marker, the AFLP can't switch to another enzyme that cut more frequently in the genome. Recently, restriction-site-associated DNA sequencing (RAD-seq) has been employed to identify sex-specific markers and describe sex determination in many species [23-29]. In Anolis carolinensis, sex-specific molecular markers identified using RAD-seq were found to be conserved in other Anolis species [27]. In Danio rerio, RAD-seq was employed to analyze F2 offspring reciprocal crosses and revealed a sex-associated locus at the end of the arm of Chr-4 in both family A (offspring of Nadia female and "AB male http://zfin.org/action/genotype/genotypedetail?zdbID=ZDBFISH-960809-7) and family B (offspring of " $\mathrm{AB}$ female and a Nadia male) as well as a locus on chr-3 in family B [24].

Chinese giant salamander Andrias davidianus is the world's largest extant amphibian and was historically widely distributed in China. However, due to the environmental degradation and human harvesting, the wild population has sharply decreased, and it is now classified as an endangered species. A.davidianus possesses 30 pairs of chromosomes with 19 pairs of microchromosomes [18] and a genome of $250 \mathrm{~GB}$, making assembly a challenge [30]. Therefore, RAD-seq is a useful method to explore a sex-specific marker in A. davidianus.

The goal of this study was to identify a sex-specific genetic marker in A. davidianus through the RAD-seq. We firstly used the female RAD-seq data to check against the male RAD-seq data and produced the putative female specific sequences, and then the putative female specific sequences were blasted against the male genome data and produced the candidate sex specific sequences. The candidate sex specific marker was validated by comparing to individuals with sex identified by PCR amplification. These markers were used to identify the sex reversal salamander from the individuals exposed to high temperature or sex hormone.

\section{Methods}

\section{RAD library construction and sequencing}

Two male (X1, X2) and two female (C1, C2) one-year-old, apparently healthy, $A$. davidianus were obtained from Zhejiang Yongqiang Chinese Giant Salamander Ltd. (Jinhua, Zhejiang Province, China). An additional two male (X3, X8) and female (C3, C8) adult A. davidianus were obtained from Shandong Yimeng Chinese Giant Salamander Ltd. (Yimeng, Shandong Province, China) (Table 1). The individuals were killed after anesthesia with MS222 according to Yangtze River Fisheries Research Institute Care Committee (No. 2013001). Physiological sex was determined by histology. Genomic DNA was extracted using the TIANamp Genomic DNA Kit (Tiangen, Beijing, China) including RNase A treatment, and the concentration and quality was detected by Agilent 2100 Bioanalyzer (Agilent Technologies, Santa Clara, CA) and agarose gel electrophoresis. Genomic DNA was double-digested using restriction enzymes EcoRI and NlaIII (New England Biolabs) following the conditions $1 \mu \mathrm{l}$ of EcoRI-HF (Fermantas, 20 units), $1 \mu$ of NIAIII (Fermantas, 20 units), $5 \mu \mathrm{l}$ of Fermantas buffer, $25 \mu \mathrm{l}$ of DNA (500 ng of DNA), and double-distilled water to a final volume of $50 \mu \mathrm{l}$. The reaction was incubated at $37^{\circ} \mathrm{C}$ for $1 \mathrm{~h}$ and subsequently at $65{ }^{\circ} \mathrm{C}$ for $30 \mathrm{~min}$ to inactivate the restriction enzyme. The resulting fragment was purified by MiniElut DNA-Pure Kit (Sangon Biotech, Shanghai, China) and ligated to P1 adapters with EcoRI restriction sites and the P2 adapter binding to overhangs generated by NIAIII. Each reaction used $20 \mu \mathrm{l}$ (200 ng) of digested genomic DNA, $2 \mu \mathrm{l}$ of T4 ligation buffer (NEB), $5 \mu \mathrm{l}$ of adapters $(0.02 \mu \mathrm{M}$ Adapter P1 $=0.1 \mathrm{pmol}, 3 \mu \mathrm{M}$ Adapter P2 $=15$ pmol), $0.5 \mu$ l of T4 ligase (NEB, 200 units), $4 \mu$ ATP 10 $\mathrm{mM}$, and double-distilled water to a final volume of $40 \mu \mathrm{l}$. The ligation was performed via polymerase chain reaction (PCR) at $37^{\circ} \mathrm{C}$ for $30 \mathrm{~min}$ followed by $65^{\circ} \mathrm{C}$ for $30 \mathrm{~min}$.

Following ligation to the adapters, DNA samples were cleaned using the QIAGEN QIAquick PCR Purification Kit, and PCR was carried out to replicate the cleaned fragments. Each PCR reaction contained $10 \mu \mathrm{l}$ of purified fragments, $10 \mu \mathrm{l}$ of $5 \mathrm{x}$ NEB Master Mix, $2 \mu \mathrm{l}$ of primer $(10 \mu \mathrm{m}$ Illumina PE), and $28 \mu \mathrm{l}$ of double-distilled water 
Table 1 Sample sequenced using RAD-Seq and summary of RAD -seq analyses

\begin{tabular}{|c|c|c|c|c|c|c|c|c|c|}
\hline Sample & location & Reads & $\begin{array}{l}\text { Number of base } \\
\text { pairs }\end{array}$ & MID & RAD-tag & $\begin{array}{l}\text { Mean Depth of RAD- } \\
\text { tag }\end{array}$ & $\begin{array}{l}\text { Sequence } \\
\text { coverage }\end{array}$ & $\begin{array}{l}\text { Q20 } \\
(\%)\end{array}$ & $\begin{array}{l}\text { Q30 } \\
(\%)\end{array}$ \\
\hline \multicolumn{10}{|l|}{ Female } \\
\hline $\mathrm{adCl}$ & Zhejiang & $66,274,208$ & $9,531,810,907$ & GCTAC & $6,507,944$ & 10.2 & 0.19 & 97.92 & 93.56 \\
\hline $\operatorname{adC2}$ & Zhejiang & $139,991,345$ & $20,139,630,928$ & ССТCT & $9,766,767$ & 14.3 & 0.41 & 97.94 & 93.59 \\
\hline $\operatorname{adC3}$ & Shandong & $155,877,412$ & $22,421,346,688$ & TAATC & $10,029,072$ & 15.54 & 0.44 & 97.93 & 93.58 \\
\hline $\operatorname{adC} 8$ & Shandong & $93,172,870$ & $13,367,981,397$ & $\begin{array}{l}\mathrm{GGCT} \\
\mathrm{AC}\end{array}$ & $8,583,073$ & 10.9 & 0.27 & 97.91 & 94.17 \\
\hline Subtotal & - & $455,315,835$ & $65,460,769,920$ & - & $34,886,856$ & 50.94 & 1.31 & - & - \\
\hline Subaverage & - & $113,828,958$ & $16,365,192,480$ & - & $8,721,714$ & 12.73 & 0.33 & - & - \\
\hline \multicolumn{10}{|l|}{ Male } \\
\hline $\operatorname{adX1}$ & Zhejiang & $127,979,232$ & $18,418,604,178$ & GCTTA & $9,167,503$ & 13.9 & 0.37 & 97.97 & 93.7 \\
\hline $\operatorname{adX2}$ & Zhejiang & $204,881,740$ & $29,486,474,863$ & TCCAC & $11,078,999$ & 18.5 & 0.59 & 97.98 & 93.7 \\
\hline $\mathrm{adX3}$ & Shandong & $66,176,492$ & $9,590,086,571$ & CTCC & $6,443,154$ & 10.2 & 0.20 & 97.93 & 93.56 \\
\hline $\operatorname{adX} 8$ & Shandong & $79,719,690$ & $11,421,575,816$ & АCCTCT & $8,038,551$ & 9.9 & 0.23 & 97.95 & 94.1 \\
\hline Subtotal & - & $478,757,154$ & $68,916,741,428$ & - & $34,728,207$ & 52.5 & 1.02 & - & - \\
\hline Subaverage & - & $119,689,288$ & $17,229,185,357$ & - & $8,682,051$ & 13.13 & 0.26 & - & - \\
\hline Total & - & $934,072,989$ & $134,377,511,348$ & - & $69,615,063$ & 103.44 & 2.33 & - & - \\
\hline Average & - & $116,759,123$ & $16,797,188,918$ & - & $8,701,882$ & 12.93 & 0.29 & - & - \\
\hline
\end{tabular}

to a final volume of $50 \mu \mathrm{l}$. PCR conditions were $30 \mathrm{~s}$ at $95^{\circ} \mathrm{C}$ followed by 16 cycles of $30 \mathrm{~s}$ at $95^{\circ} \mathrm{C}, 20 \mathrm{~s}$ at $62^{\circ} \mathrm{C}$, $30 \mathrm{~s}$ at $68^{\circ} \mathrm{C}$, and $5 \mathrm{~min}$ extension at $72^{\circ} \mathrm{C}$ and stored at $4{ }^{\circ} \mathrm{C}$ forever. Gel electrophoresis was performed, and fragments ranging from 400 to $600 \mathrm{bp}$ including the 75 bp adapter were excised from the gel, and QIAGEN QIAquick PCR Purification Kit was used to clean the fragments. The samples were sequenced on an Illumina HiSeq PE150 using $150 \mathrm{bp}$ paired-end reads. The sequence was deposited in the NCBI databank (SRP159124).

\section{Determining sex-specific markers}

Reads containing low quality sequence scores, adaptor sequences, missing restriction site sequences, and reads with $>10 \%$ unknown bases were removed using Trimmomatic v. 0.32 [31], and raw reads were trimmed to 110 nucleotides, which ensured that more than $98 \%$ of the nucleotides had a quality value greater than Q30. The generated reads were sorted into loci using Stacks software [32]. The reads from randomly selected individuals, $\mathrm{X} 8$ and $\mathrm{C} 8$, were assembled as a reference genome by SoapDenovo2 [33]. The assembled sequences were screened against opposite sex raw reads with Stacks v.1.46 [32, 34] (http://catchenlab.life.illinois.edu/stacks/) using the MID sequence to identify exact matches to the110 bp trimmed RAD-tag. The putative female sequences and the male sequences were checked against the male genomic data (incomplete genome data). The unmapped sequence from the female data files was regarded as the candidate female-specific marker (Fig. 1).

Validation and application of the sex-specific marker Gonads of all specimens were fixed in $4 \%$ paraformaldehyde ( $\mathrm{pH} 7.5$ ) for $24 \mathrm{~h}$, stored in $70 \%$ ethanol, dehydrated through an ethanol gradient, cleared in xylene, embedded in paraffin, and 5-6 $\mu \mathrm{m}$ sections were cut and stained with hematoxylin-eosin. The phenotypic sex was determined under light microscopy (Olympus). The genomic DNA was extracted by a DNA extraction kit (TianGen, Beijin, China) and the quality evaluated by agarose gel electrophoresis and NanoPhotometer-N50 (Implen, Germany). One-hundred pairs of primers were designed according to the sequences of the putative female-specific markers by Primer Premier 3.0 (Addtional file 2: Table S1). Polymerase chain reaction reaction was carried out on $7.5 \mu \mathrm{l} 2 \times$ PCR Mix (Dongsheng Biotech, Guangdong, China), $0.2 \mu \mathrm{l} 10 \mathrm{uM}$ forward/reverse primer, $1 \mu \mathrm{l}(50-100 \mathrm{ng} / \mu \mathrm{l})$ genomic DNA, and doubled-distilled $\mathrm{H}_{2} \mathrm{O}$ to a volume of $15 \mu \mathrm{l}$. Reaction conditions were $94^{\circ} \mathrm{C}$ for 5 min followed by 35 cycles of $94{ }^{\circ} \mathrm{C}$ for $30 \mathrm{~s}$, primer-specific temperature for $30 \mathrm{~s}$, and $72{ }^{\circ} \mathrm{C}$ for $40 \mathrm{~s}$ with a final extension at $72{ }^{\circ} \mathrm{C}$ for $5 \mathrm{~min}$. The putative female-specific markers were initially validated on five male and five female individuals, and further validation was performed on 24 males and 24 females. From a previous study, we collected 12 females 
a

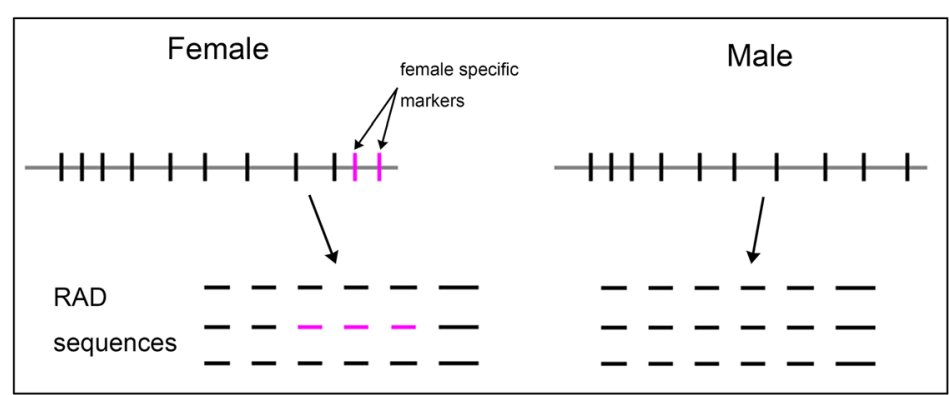

b

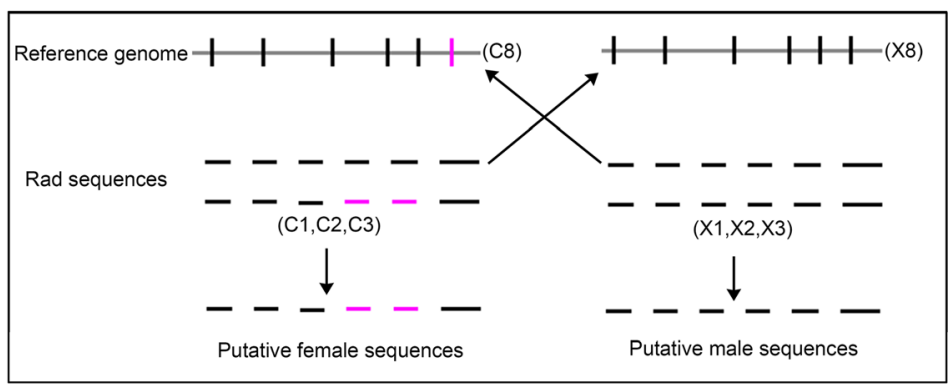

c

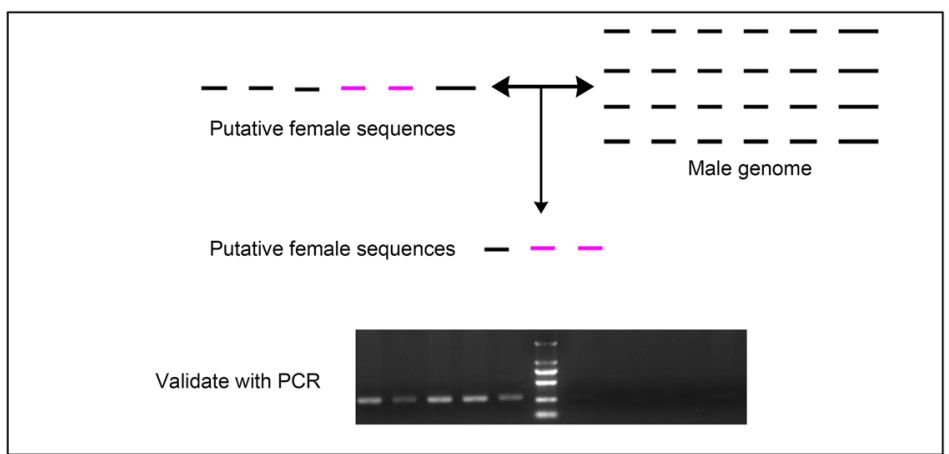

Fig. 1 workflow of the female specific marker identification: a. locations of restriction sites along chromosomes in male and female specimens and RAD sequences; $\mathbf{b}$. bioinformatic analysis of the RADseq libraries; $\mathbf{c}$. further analysis of the putative specific marker and validation with PCR

and 12 males after high temperature $\left(28^{\circ} \mathrm{C}\right)$ exposure and 20 females and 3 males after $17 \beta$-estradiol exposure at concentration of $500 \mu \mathrm{g} / \mathrm{l}$. The genetic sex was determined by the validated sex-specific marker.

\section{Results}

\section{Restriction site-associated DNA sequencing and}

\section{assembling}

The RAD-Seq of four male and four female A. davidianus was performed on an Illumina HiSeq PE150 sequencing platform, and 93.4 million reads and 134,377,511,348 bp of data were generated. The male RAD-seq library contained 47.8 million reads with $68,916,741,428 \mathrm{bp}$ of data, and the female RAD-seq library produced 45.5 million reads with $65,460,769,920 \mathrm{bp}$ of data, a mean of 11.6 million reads and 16,797,188,918 bp for each salamander. A total of 69,615,063 RAD-tags was used in the study $(34,886,856$ female and $34,728,207$ male) with a mean of $8,701,882$ per salamander. The number of the RAD-tags recovered from each specimen ranged from 6,443,154 to $11,078,999$ and strongly correlated with the reads recovered from each individual, $66,176,492$ to $204,881,740 \mathrm{bp}$ (Table 1). The mean depth of the RAD-tag ranged from 10.2 to 15.54 in female groups and from 9.9 to 18.5 in male groups (Table 1). According to the genome size, the sequence coverage ranged from $0.19-0.44 \mathrm{x}$ in female groups and $0.20-0.59 \mathrm{x}$ in male groups. The average sequence coverage was $0.29 \times$ per individual.

A total of 2,663,744 scaffolds in X8 and 2,949,503 in C8 were assembled with $453,211,396 \mathrm{bp}$ and $584,940,914 \mathrm{bp}$, respectively. The greatest lengths were $1572 \mathrm{bp}$ in X8 and $1987 \mathrm{bp}$ in C8, and the number of scaffolds $>1000 \mathrm{bp}$ was 67 in X8 and 176 in C8. The N50 length was 148 bp in X8 and $144 \mathrm{bp}$ in C8 (Table 2).

\section{Validation of the sex-specific markers}

Phenotypic sex was determined by examination of gonads (Additional file 1). Female assembled sequences 
Table 2 Summary of the results for the assembly

\begin{tabular}{lll}
\hline ID & X8 & C8 \\
\hline Scaffold Number & $2,663,744$ & $2,949,503$ \\
Large scaffolds (> 1000 bps) & 65 & 176 \\
Greatest length (bp) & 1572 & 1987 \\
N50 length (bp) & 148 & 144 \\
N90 length (bp) & 144 & 144 \\
GC content (\%) & 46.38 & 46.46 \\
Total base pairs (bp) & $453,211,396$ & $584,940,914$ \\
\hline
\end{tabular}

screened against the raw reads of male files (X1, X2, X3) yielded 19,097 female sequences, and 17,994 male sequences after the male assembled sequences for the female raw read files $(\mathrm{C} 1, \mathrm{C} 2, \mathrm{C} 3)$. A further BLAST search was carried out between unmatched sequences and the male genome. Three-hundred-eight female sequences and 542 male sequences were unmapped to the male genome. One-hundred unmapped female sequences were randomly selected, primers were designed according the sequences (Additional file 2), and four female sequences were filtered out for initial validation (Table 3). We defined sequences according to their length as adf431, adf340, adf318, and adf225. The four female sequences exhibited four specific bands in the 24 female specimens and no band in the male (Fig. 2). BLAST searches of the four female-specific sequences in GenBank revealed no high homology sequence.

\section{Application of the sex-specific markers}

Twelve males and twelve females exposed to high temperature were examined for the female-specific marker adf340, and all females showed the expected band, while three males displayed a similar band and were identified as genetic female reversed to physiological male (Fig. 3). Twenty females and 3 males exposed to $17 \beta$-estradiol were examined by the female-specific marker adf431. Three males and one negative control showed no specific band, and 13 of 20 females displayed no band and were identified as genetic male reversed to physiological female (Fig. 3).

\section{Discussion}

In the recent years, RAD-seq technology was widely used to develop the molecular marker in species. In eight salamanders, $134.38 \mathrm{~Gb}$ of data were generated by RAD-seq with mean data per salamander of $16.79 \mathrm{~Gb}$. In Pistacia vera, $36.96 \mathrm{~Gb}$ of data was sequenced from 18 plants with a mean of $2.05 \mathrm{~Gb}$ per plant to identify the sex-linked SNP marker [28]. In Lupinus angustifolius, $17.33 \mathrm{~Gb}$ data from 20 plants produced $0.87 \mathrm{~Gb}$ per plant $[35,36]$. In Anolis carolinensis, 19.9 million reads generated by RAD-seq and 51, 438 RAD-tag from 17 individuals, recovering RAD-tag per individual from 23,524 to 44,408 [27]. We obtained higher total and mean data per specimen than reported in Anolis carolinensis, Lupinus angustifolius, or Pistacia vera. Due to the large genome of $A$. davidianus ( $50 \mathrm{~Gb}$ ) analyzed by flow cytometry [30], the sequence coverage in this study averaged $0.29 \mathrm{x}$ per salamander. The results were available to explore the sex-specific marker to identify the genetic sex of $A$. davidianus, especially the larvae and sex reversed individuals in the non-model species.

This study was one of few to use RAD-seq to develop sex-specific markers without a genetic linkage map. In Anolis carolinensis, sex-specific molecular markers were explored using RAD-seq in seven males and ten females, the accuracy was tested by PCR, and male heterogamety was further confirmed by the sex specific marker [27]. A study of Pistacia vera identified sex-linked SNP markers, and the sex determination mechanism was identified as the ZZ/ZW type using the RAD-seq [28]. In other species, the sex-linked marker has been explored using the genetic linkage map by RAD-seq. In Nile tilapia Oreochromis niloticus, RAD-seq was used to compare the SNP marker in pseudomales and females, and association analysis with a set of SNPs confirmed that the genomic region of LG23 exerts a significant effect on temperature-dependent sex [37]. In Polyprion oxygeneios, a genetic linked group was constructed, and a

Table 3 Female-specific primers

\begin{tabular}{llll}
\hline Primer & Sequence $\left(5^{\prime}-3^{\prime}\right)$ & Annealing temperature $\left({ }^{\circ} \mathrm{C}\right)$ & Product size $(\mathrm{bp})$ \\
\hline adf225s & CCATGCCCTGTACATTGCG & 59.899 & 162 \\
adf225a & CCGTGAACATGGAGGGGTT & 60.251 & 251 \\
adf340s & TTAACGGCCCTAACACCAGG & 59.674 \\
adf340a & GGTTAGGGCGGCTCTGATT & 60.107 \\
adf318a & TATGTCAGGGTGATCAAACTCTTCA & 59.5 \\
adf318s & CTAGAAGACGTGGTGCCATG & 60.0 \\
adf431a & TCCAGAATGAAGTCCTGGCCT & 59.1 \\
adf431s & CGAGCCTCCATTGTGCCTT & 59.8 & 266 \\
\hline
\end{tabular}



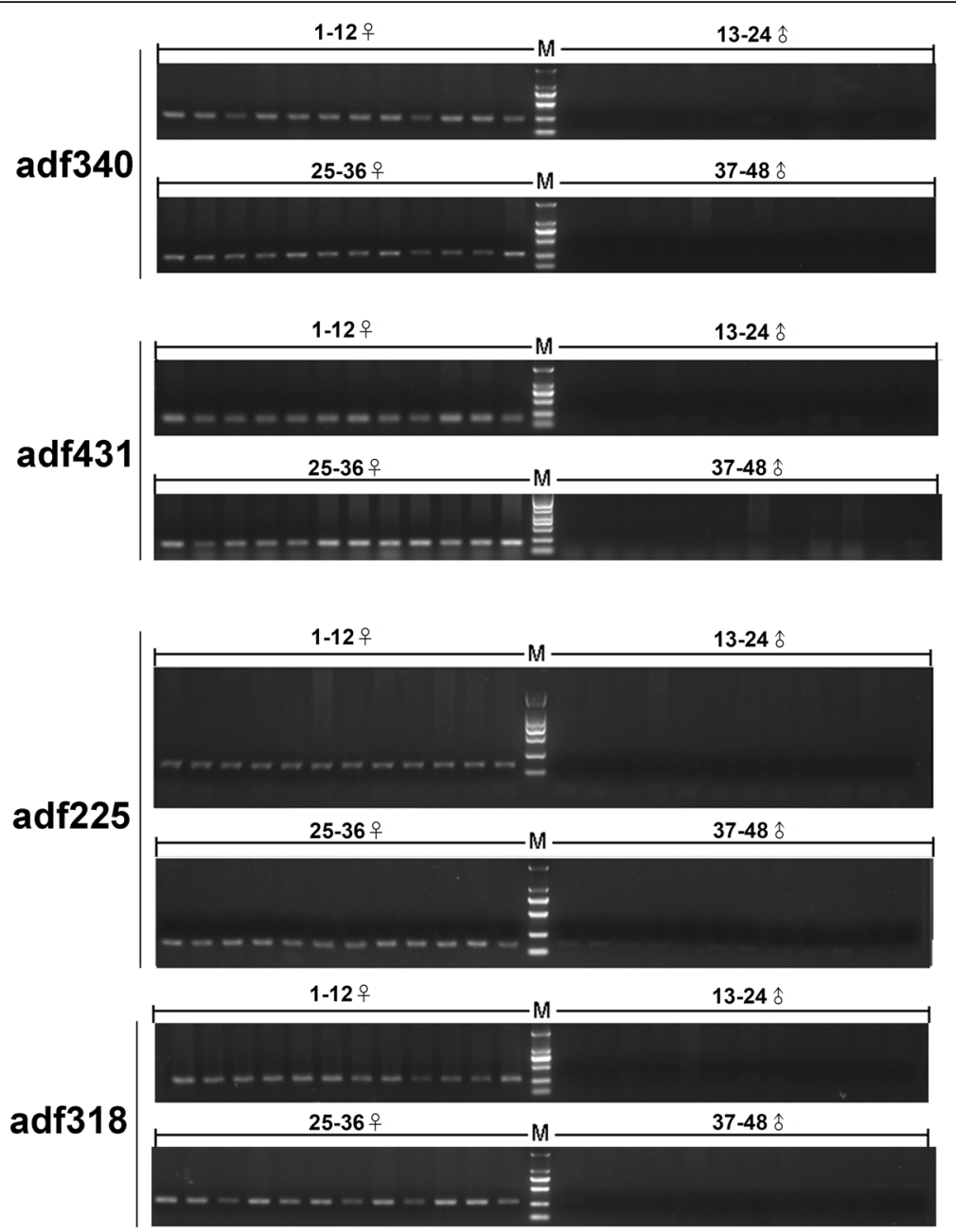

Fig. 2 Validation of four female-specific markers in 24 female and 24 male Andrias davidianus

single major sex-determining locus was mapped to LG 14. Several markers were found to be strongly linked to the sex-determining locus [38]. In Gadus morhua, malespecific region of $9 \mathrm{~kb}$ was mapped on linkage group 11 annotating a single gene named $\mathrm{zkY}$ on the $\mathrm{Y}$ chromosome. Expression of zkY was high level in the developing larvae before the onset of sex differentiation [39]. In Rana clamitans, 13 sex-linked SNP loci and eight loci associated with males were identified by Diversity Arrays Technology [40, 41], which employs a combination of genome complexity reduction and next-generation sequencing similar to RAD-seq and genotyping-by-sequencing methods [42]. RAD-seq is an effective means of defining a molecular marker.

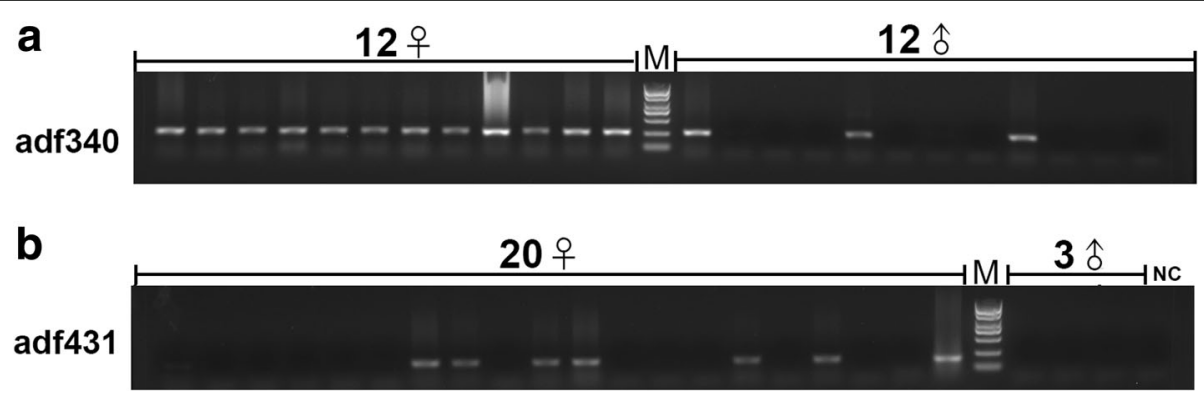

Fig. 3 Female-specific marker used to identify sex reversal in Andrias davidianus exposed to high temperature or 17ß-estradiol. NC, negative control 
Using sex-specific sequences to identify genetic sex or the sex chromosome shows advantages over cytogenetics. Cytogenetic methods are not possible in species possessing microchromosomes or lacking a heteromorphic sex chromosome, such as the majority of amphibians, reptiles, and fish [19, 43-45]. A. davidianus has been reported to possess 30 pairs of chromosomes including 19 pairs of telocentric chromosomes [18], enabling identification of a sex-specific marker.

We isolated the female-specific marker and used it to reveal the genetic sex. We identified three males reversed from genetic females exposed to high temperature and 13 females reversed from genetic males exposed to $17 \beta$-estradiol. These results agreed with our previous study in which sex reversed individuals were identified by a different sex-specific marker [46], suggesting that these sex specific-markers are highly consistent and valid. In Pelteobagrus fulvidraco, Y- and X-linked markers were isolated and used to identify sex reversed individuals and YY super-males to produce the all-male population [3]. In Pseudobagrus ussuriensis, male-specific sequences were employed to identify genetic sex, suggesting male heterogametic sex determination [47]. A sex-specific sequence was used to identify genetic sex and the WW super female in Cynoglossus semilaevis [20, 21]. A sex-specific marker was used to genotype Hyla arborea and showed that all females were homozygous for allele 235 , while the males were heterozygous (235/241), suggesting a male heterogamety sex determination system [48].

Results of the present study have implications for both fundamental and applied research: First, the sex-specific marker can be used to identify the sex determination system, which will help describe the evolution of sex determination in amphibians. Second, the sex specific marker allows investigation of parent influence on offspring sex ratio. Third, identification of the sex-specific marker has potential value for conservation biology. Climate change has been shown to affect the sex ratio of the green sea turthe Chelonia mydas, in the Southwest Pacific, and warm northern Great Barrier Reef nesting beaches were female-biased at $99.1 \%$ of juveniles, $99.8 \%$ of subadults, and $86.8 \%$ of adults [49]. In order to protect C. mydas from extinction due to complete feminization, male specimens were identified and released. Similarly, the A. davidianus sex specific marker could allow release into the wild at the optimum sex ratio.

\section{Additional files}

Additional file 1: Histology sections of the gonads of Andrias davidianus. Female gonad; B. Male gonad. GrC: Granulosa cells; GC: Germ cell; FC: Follicular cavity; SL: seminiferous lobule; SC: somatic cell. (TIF $7268 \mathrm{~kb})$

Additional file 2: Primers used. (DOCX $35 \mathrm{~kb}$ )

\section{Abbreviations}

AFLP: Amplified fragment length polymorphism; Chr-4: Chromosome 4; LG: Link group; PCR: Polymerase chain reaction; RAD: Restriction siteassociated DNA sequencing; SNP: Single nucleotide polymorphisms

\section{Acknowledgements}

We acknowledge the assistance for data analysis by LinEn laboratory.

\section{Funding}

This work was supported by National Nature Science Foundation of China (31502155), Key Laboratory of Freshwater Aquatic Biotechnology and Breeding, Ministry of Agriculture, Heilongjiang Fisheries Research Institute, Chinese Academy of Fishery Sciences (KF-2017-06). The funding body did not play any role in the design of the study and collection, analysis, and interpretation of data and in writing the manuscript.

\section{Availability of data and materials}

All data generated or analyzed during this study are included in this article and its supplementary information files.

\section{Authors' contributions}

$\mathrm{QMH}, \mathrm{HBX}$, and CSX conceived and designed the experiments; QMH, CFC, HFT, ZGQ, and YM analyzed the data; QHW, LW, and QMH collected the samples and performed the experiments; $\mathrm{QMH}$ and $\mathrm{HBX}$ wrote the paper. All authors reviewed the manuscript. All authors read and approved the final manuscript.

\section{Authors' information}

Q.M. Hu., Q.H.W., H.F.T., Y.M., H.B.X., Yangtze River Fisheries Research Institute, Chinese Academy of Fishery Sciences, Wuhan, Hubei 430223, China; C.F. C., C.S.X., State Key Laboratory Cultivation Base for Cell Differentiation Regulation, College of Life Science, Henan Normal University, Xinxiang 453007, Henan Province, China; Q.H.W., College of Life Science, Yangtze University, Jingzhou 434025, China; Z.G.Q., L.W., College of Fisheries, Henan Normal University, Xinxiang 453007, China.

\section{Ethics approval}

The cultivated salamander used in the study was permitted by the animal owners who agreed to be involved in the project. Handling and husbandry of salamander and other relevant protocols used in this study were approved by Yangtze River Fisheries Research Institute Care Committee protocols 2013001. The methods used in this study were performed in strict accordance with the Laboratory Animal Management Principles of China.

\section{Consent for publication}

Not applicable.

\section{Competing interests}

The authors declare that they have no competing interests.

\section{Publisher's Note}

Springer Nature remains neutral with regard to jurisdictional claims in published maps and institutional affiliations.

\section{Author details \\ ${ }^{1}$ Yangtze River Fisheries Research Institute, Chinese Academy of Fishery Sciences, Wuhan 430223, Hubei, China. ${ }^{2}$ State Key Laboratory Cultivation Base for Cell Differentiation Regulation, College of Life Science, Henan Normal University, Xinxiang 453007, Henan Province, China. ${ }^{3}$ College of Life Science, Yangtze University, Jingzhou 434025, China. ${ }^{4}$ College of Fisheries, Henan Normal University, Xinxiang 453007, China.}

Received: 12 October 2018 Accepted: 3 May 2019

Published online: 23 May 2019

References

1. Nakamura M. Sex determination in amphibians. Semin Cell Dev Biol. 2009; 20:271-82.

2. Flament S. Sex reversal in amphibians. Sex Dev. 2016;10(5-6):267-78. 
3. Wang D, Mao HL, Chen HX, Liu HQ, Gui JF. Isolation of Y- and X-linked SCAR markers in yellow catfish and application in the production of all-male populations. Anim Genet. 2009;40:978-81.

4. Nakayama I. Sex control research for aquatic animal breeding. J Anim Genet. 2010;31:57-66.

5. Chen SL, Deng SP, Ma HY, Tian YS, Xu JY, Yang JF, et al. Molecular markerassisted sex control in half-smooth tongue sole (Cynoglossus semilaevis). Aquaculture. 2008;283:7-12.

6. Altmanová M, Rovatsos M, Kratochvíl L, Johnson Pokorná M. Minute Y chromosomes and karyotype evolution in Madagascan iguanas (Squamata: Iguania: Opluridae). Biol J Linn Soc. 2016;118:618-33.

7. Šíchová J, Ohno M, Dincă V, Watanabe M, Sahara K, Marec F. Fissions, fusions, and translocations shaped the karyotype and multiple sex chromosome constitution of the northeast-Asian wood white butterfly, Leptidea amurensis. Biol J Linn Soc. 2016:118:457-71.

8. Ponse K. Sur la digametic du crapaud hermaphrodite. Rev Suisse Zool. 1942; 49:185-9.

9. Chang $C Y$, Witschi E. Gene control and hormonal reversal of sex differentiation in Xenopus. Proc Soc Exp Biol Med. 1956;93:140-4.

10. Humphrey RR. Male homogametry in the Mexican axolotl: a study of the progeny obtained when germ cells of a genetic male are incorporated in a developing ovary. J Exp Zool. 1957;134:91-101.

11. Muller-Belecke A, Horstgen-Schwark G. Sex determination in Oreochromis niloticus sex ratios in homozygous gynogenetic progeny. Aquaculture. 1995; 137:103-8.

12. Hamaguchi S, Toyazaki Y, Shinomiya A, Sakaizumi M. The XX-XY sexdetermination system in Oryzias luzonensis and $O$. mekongensis revealed by the sex ratio of the progeny of sex-reversed fish. Zool Sci. 2004;21:1015-8.

13. Müller-Belecke A, Hoerstgen-Schwark G. Sex determination in Tilapia (Oreochromis niloticus). Sex ratios in homozygous gynogenetic progeny and their offspring. Aquaculture. 1995;137:57-65.

14. Piferrera F, Cal RM, Gómez C, Álvarez-Blázquez B, Castro J, Martinez P. Induction of gynogenesis in the turbot (Scophthalmus maximus):: effects of UV irradiation on sperm motility, the Hertwig effect and viability during the first 6 months of age. Aquaculture. 2004;238:403-19.

15. Ezaz MT, Myers JM, Powell SF, Mcandrew BJ, Penman DJ. Sex ratios in the progeny of androgenetic and gynogenetic YY male Nile tilapia, Oreochromis niloticus L. Aquaculture. 2004;232:205-14.

16. Tvedt HB, Benfey TJ, Martinrobichaud DJ, Mcgowan C, Reith M. Gynogenesis and sex determination in Atlantic halibut (Hippoglossus hippoglossus). Aquaculture. 2006;252:573-83.

17. Li XY, Liu XL, Zhu YJ, Zhang J, Ding M, Wang MT, et al. Origin and transition of sex determination mechanisms in a gynogenetic hexaploid fish. Heredity. 2018;121:64-74.

18. Zhu B, Feng Z, Qu A, Gao H, Zhang Y, Sun D, et al. The karyotype of the caudate amphibian Andrias davidianus. Hereditas. 2002;136:85-8.

19. Ezaz T, Sarre S, O'Meally D, Graves J, Georges A. Sex chromosome evolution in lizards: independent origins and rapid transitions. Cytogenet Genome Res. 2009;127:249-60.

20. Chen SL, Li J, Deng SP, Tian YS, Wang QY, Zhuang ZM, et al. Isolation of female-specific AFLP markers and molecular identification of genetic sex in half-smooth tongue sole (Cynoglossus semilaevis). Mar Biotechnol. 2007;9: 273-80

21. Chen SL, Ji XS, Shao CW, Li WL, Yang JF, Liang Z, et al. Induction of mitogynogenetic diploids and identification of WW super-female using sexspecific SSR markers in half-smooth tongue sole (Cynoglossus semilaevis). Mar Biotechnol. 2012;14:120-8.

22. Chen X, Mei J, Wu J, Jing J, Ma W, Zhang J, Dan C, Wang W, Gui JF. A comprehensive transcriptome provides candidate genes for sex determination/differentiation and SSR/SNP markers in yellow catfish. Mar Biotechnol. 2015;17:190-8.

23. Baird NA, Etter PD, Atwood TS, Currey MC, Shiver AL, Lewis ZA, et al. Rapid SNP discovery and genetic mapping using sequenced RAD markers. PLOS One. 2008:3(10):e3376.

24. Anderson JL, Rodríguez Marí A, Braasch I, Amores A, Hohenlohe P, Batzel P, et al. Multiple sex-associated regions and a putative sex chromosome in zebrafish revealed by RAD mapping and population genomics. PLoS One. 2012;7(7):e40701.

25. Palaiokostas C, Bekaert M, Khan MG, Taggart JB, Gharbi K, McAndrew BJ, et al. Mapping and validation of the major sex-determining region in Nile tilapia (Oreochromis niloticus L.) using RAD sequencing. PLoS One. 2013;8:e68389.
26. Carmichael SN, Bekaert M, Taggart JB, Christie HR, Bassett DI, Bron JE, et al. Identification of a sex-linked SNP marker in the Salmon louse (Lepeophtheirus salmonis) using RAD sequencing. PLoS One. 2013;16(8):e77832.

27. Gamble T, Zarkower D. Identification of sex-specific molecular markers using restriction site-associated DNA sequencing. Mol Ecol Resour. 2014;14:902-13.

28. Kafkas S, Khodaeiaminjan M, Güney M, Kafkas E. Identification of sex-linked SNP markers using RAD sequencing suggests ZW/ZZ sex determination in Pistacia vera L. BMC Genomics. 2015;16:98.

29. Gamble T. Using RAD-seq to recognize sex-specific markers and sex chromosome systems. Mol Ecol. 2016;25:2114-6.

30. Geng X, Li W, Shang H, Gou Q, Zhang F, Zang X, et al. A reference gene set construction using RNA-seq of multiple tissues of Chinese Giant salamander Andrias davidianus. Gigascience. 2017;6:1-7.

31. Bolger AM, Lohse M, Usadel B. Trimmomatic: a flexible trimmer for Illumina sequence data. Bioinformatics. 2014:30:2114-20.

32. Catchen JM, Amores A, Hohenlohe P, Cresko W, Postlethwait JH. Stacks: building and genotyping loci de novo from short-read sequences $\mathrm{G} 3$ Genesgenetics. 2011; $1: 171-182$

33. Luo R, Liu B, Xie Y, Li Z, Huang W, Yuan J, et al. SOAPdenovo2: an empirically improved memory-efficient short-read de novo assembler. Gigascience. 2012;1:18.

34. Catchen J, Hohenlohe PA, Bassham S, Amores A, Cresko WA. Stacks: an analysis tool set for population genomics. Mol Ecol. 2013;22:3124-40.

35. Yang H, Tao Y, Zheng Z, Li C, Sweetingham MW, Howieson JG. Application of next-generation sequencing for rapid marker development in molecular plant breeding: a case study on anthracnose disease resistance in Lupinus angustifolius L. BMC Genomics. 2012;13:318.

36. Yang H, Tao Y, Zheng Z, Shao D, Li Z, Sweetingham MW, et al. Rapid development of molecular markers by next-generation sequencing linked to a gene conferring phomopsis stem blight disease resistance for marker assisted selection in lupin (Lupinus angustifolius L.) breeding. Theor Appl Genet. 2013;126:511-22.

37. Wessels S, Krause I, Floren C, Schütz E, Beck J, Knorr C. ddRADseq reveals determinants for temperature-dependent sex reversal in Nile tilapia on LG23. BMC Genomics. 2017;18:531.

38. Brown JK, Taggart JB, Bekaert M, Wehner S, Palaiokostas C, Setiawan A. Et al. mapping the sex determination locus in the hāpuku (Polyprion oxygeneios) using ddRAD sequencing. BMC Genomics. 2016;17:448.

39. Kirubakaran TG, Andersen $\varnothing$, De Rosa MC, Andersstuen T, Hallan K, Kent MP, Lien S. Characterization of a male specific region containing a candidate sex determining gene in Atlantic cod. Sci Rep. 2019;9:116.

40. Kilian A, Wenzl P, Huttner E, Carling J, Xia L, Blois H, et al. Diversity arrays technology: a generic genome profiling technology on open platforms. Methods Mol Biol. 2012:888:67-89.

41. Lambert MR, Skelly DK, Ezaz T. Sex-linked markers in the north American green frog (Rana clamitans) developed using DArTseq provide early insight into sex chromosome evolution. BMC Genomics. 2016;17:844.

42. Elshire RJ, Glaubitz JC, Sun Q, Poland JA, Kawamoto K, Buckler ES, et al. A robust, simple genotyping-by-sequencing (GBS) approach for high diversity species. PLoS One. 2011;6:e19379.

43. Devlin RH, Nagahama Y. Sex determination and sex differentiation in fish: an overview of genetic, physiological, and environmental influences. Aquaculture. 2002;208:191-364.

44. Catroli GF, Kasahara S. Conserved karyotypes in cophomantini: cytogenetic analysis of 12 species from 3 species groups of Bokermannohyla (Amphibia: Anura: Hylidae). J Herpetol. 2011:45:120-8.

45. Zlotina A, Dedukh D, Krasikova A. Amphibian and avian karyotype evolution: insights from Lampbrush chromosome studies. Genes. 2017;8(11).

46. Hu Q, Tian H, Li W, Meng Y, Wang Q, Xiao H. Identification of critical sexbiased genes in Andrias davidianus by de novo transcriptome. Mol Gen Genomics. 2018. https://doi.org/10.1007/s00438-018-1508-4.

47. Pan ZJ, Li XY, Zhou FJ, Qiang XG, Gui JF. Identification of sex-specific markers reveals male heterogametic sex determination in Pseudobagrus ussuriensis. Mar Biotechnol. 2015;17:441-51.

48. Berset-Brändli $L$, Jaquiéry J, Dubey $S$, Perrin N. A sex-specific marker reveals male heterogamety in European tree frogs. Mol Biol Evol. 2006;23:1104-6.

49. Jensen MP, Allen CD, Eguchi T, Bell IP, LaCasella EL, Hilton WA, et al. Environmental warming and feminization of one of the Largest Sea turtle populations in the world. Curr Biol. 2018;28:154-9. 